\title{
Effective Encapsulation of Europium(3+) by a Heptadentate Schiff-Base Ligand
}

\begin{tabular}{|r|l|}
\hline Journal: & Zeitschrift für Anorganische und Allgemeine Chemie \\
\hline Manuscript ID: & zaac.201000141.R1 \\
\hline Diley - Manuscript type: & Communication \\
\hline Complete List of Authors: & $\begin{array}{l}\text { Edelmann, Frank; Otto-von-Guericke-Universität Magdeburg, } \\
\text { Lehrstuhl für Anorganische Chemie } \\
\text { Dröse, Peter } \\
\text { Blaurock, Steffen } \\
\text { Hrib, Cristian G. }\end{array}$ \\
\hline Keywords: & $\begin{array}{l}\text { Europium , Schiff-base ligands, TRENDSAL ligand, Heptadentate } \\
\text { ligands }\end{array}$ \\
\hline
\end{tabular}

\section{s) ScholarONE \\ Manuscript Central}


* Corresponding Author Fax: +40-391-67-18327

E-mail: frank.edelmann@ovgu.de

[a] Chemisches Institut der Otto-von-Guericke-Universität Magdeburg, Universitätsplatz 2, 39106 Magdeburg, Germany

Supporting information for this article is available on the WWW under http://dx.doi.org/10.1002/zaac.201000xxx or from the author.

\section{Introduction}

Macrocyclic Schiff-base ligands are of significant current interest as contrast media in magnetic resonance imaging $[1,2]$ and as encapsulating ligands for radiopharmaceuticals [3]. Such ligands have become very popular due to their relatively straightforward synthesis as well as their multidentate nature which results in very high binding constants for many $d$ - and $f$-block metals. In the coordination chemistry of lanthanids, steric saturation of the coordination sphere rather than electron-count is one of the guiding principles. Among the large number of ligand systems studied thus far in this respect, Schiff-base derivatives of the TREN moiety $\mathrm{N}\left(\mathrm{CH}_{2} \mathrm{CH}_{2} \mathrm{NH}_{2}\right)_{3}$ have been shown to be very effective, especially those derived from the reaction of salicylaldehyde derivatives with TREN. There have been a number of studies of the coordination chemistry of the unsubstituted parent ligand $\mathrm{N}\left[\mathrm{CH}_{2} \mathrm{CH}_{2} \mathrm{~N}=\mathrm{CH}\left(2-\mathrm{OHC}_{6} \mathrm{H}_{4}\right)\right]_{3}$ and its fully saturated analogue $\mathrm{N}\left[\mathrm{CH}_{2} \mathrm{CH}_{2} \mathrm{NHCH}_{2}\left(2-\mathrm{OHC}_{6} \mathrm{H}_{4}\right)\right]_{3}$ [4], including studies of their use as Tc-99 chelators in nuclear medicine [4b]. Orvig et al. have reported $\mathrm{Ln}$ derivatives of the saturated amino-phenol ligands $\mathrm{N}\left[\mathrm{CH}_{2} \mathrm{CH}_{2} \mathrm{NHCH}_{2}(2-\mathrm{OH}-3-\right.$ $\left.\left.\mathrm{R}^{1}-5-\mathrm{R}^{2} \mathrm{C}_{6} \mathrm{H}_{2}\right)\right]_{3}\left(\mathrm{R}^{1}=\mathrm{H}, \mathrm{OMe} ; \mathrm{R}^{2}=\mathrm{H}, \mathrm{Cl}, \mathrm{Br}\right)[5,6]$, and mixed-metal and single-metal lanthanide derivatives of a range of Schiff-base ligands of the type $\mathrm{N}\left[\mathrm{CH}_{2} \mathrm{CH}_{2} \mathrm{~N}=\mathrm{CH}(2-\right.$ $\left.\left.\mathrm{OH}-3-\mathrm{R}^{1}-5-\mathrm{R}^{2} \mathrm{C}_{6} \mathrm{H}_{2}\right)\right]_{3}$ have also been reported [6-14].

The di-tert-butyl substituted analogue $\mathrm{N}\left[\mathrm{CH}_{2} \mathrm{CH}_{2} \mathrm{~N}=\mathrm{CH}\right.$ (2-OH-3,5- $\left.\left.{ }^{\mathrm{t}} \mathrm{Bu}_{2} \mathrm{C}_{6} \mathrm{H}_{2}\right)\right]_{3} \quad$ (= TRENDSAL) was previously employed for the rare earth elements $\mathrm{Gd}, \mathrm{Sm}$, and $\mathrm{Nd}$ as well as indium, leading in all cases to the formation of

mononuclear complexes [15]. This very bulky tripodal ligand is generally assumed to encapsulate even the largest lanthanide ions and thereby prevent solvation of the resulting complexes. We report here a positive proof of this assumption by showing that in the newly synthesized europium(III) complex Eu(TRENDSAL) (1) not even the "slim" ligand acetonitrile is able to enter the coordination sphere of the tightly encapsulated $\mathrm{Eu}^{3+}$ ion.

\section{Results and Discussion}

Previously reported synthetic routes to lanthanide TRENDSAL complexes involved either treatment of the lanthanide silylamide precursors $\mathrm{Ln}\left[\mathrm{N}\left(\mathrm{SiMe}_{3}\right)_{2}\right]_{3}$ with the free ligand [15a] or, in the case of $\mathrm{Gd}$, reaction of the ligand with $\mathrm{Gd}\left(\mathrm{NO}_{3}\right)_{3} \cdot 6 \mathrm{H}_{2} \mathrm{O}$ in the presence of $\mathrm{NaOH}$. [15b] The first method requires the preparation of the $\mathrm{Ln}\left[\mathrm{N}\left(\mathrm{SiMe}_{3}\right)_{2}\right]_{3}$ precursors, while the latter reaction suffers from fairly low yields $(<50 \%)$. We prepared the hitherto unknown europium(III) complex Eu(TRENDSAL) (1) by a simple one-pot reaction as illustrated in Scheme 1 via treatment of europium(III)-trichloride hexahydrate with 3,5-di-tertbutylsalicylicaldehyde and tris(2-aminoethyl)amine in a molar ratio of $1: 3: 1$. The reaction was carried out in refluxing methanol using triethylamine as $\mathrm{HCl}$-scavenger.

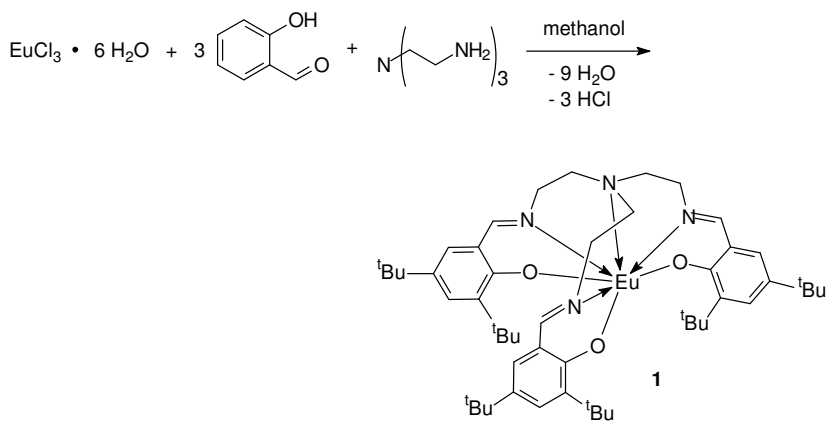

Scheme 1. Synthesis of Eu(TRENDSAL) (1). 
Soxhlet extration of the crude product with toluene afforded the desired unsolvated product as a bright yellow, crystalline solid in $71 \%$ yield.Spectroscopic characterization of unsolvated 1 was straightforward. A mass spectrum showed the molecular ion at $\mathrm{m} / \mathrm{z}, 944.7$ with $90 \%$ relative intensity. Despite the paramagnetic nature of the cerium(3+) ion a meaningful ${ }^{1} \mathrm{H}$ NMR spectrum could be obtained. Although spread out over a range of $c a .60 \mathrm{ppm}$, all signals could be unambiguously assigned.

In order to test the possibility (or impossibility) of adduct formation with donor solvents, compound $\mathbf{1}$ was recrystallized either from $\mathrm{DME}(=1,2$-dimethoxyethane) or acetonitrile. In both cases, bright yellow single crystals were obtained which were structurally characterized by X-ray diffraction. Crystallization from DME afforded the DME solvate 1·DME. Crystal data and structure refinement details are listed in Table 1, and selected bond lengths and angles for 1.DME are summarized in Table 2. As expected, the DME is not coordinated to europium but intercalated in the crystal lattice. The molecular structure of Eu(TRENDSAL) in 1.DME is depicted in Figure 1. The overall coordination geometry around the central europium(3+) ion can be best described as distorted capped octahedral with TRENDSAL acting as triply deprotonated heptadentate ligand. It is easily recognized how the very bulky TRENDSAL ligand wraps around the central $\mathrm{Eu}(3+)$ ion. The six tert-butyl substituents in the periphery of the ligand largely add to the effective shielding of the europium. The Eu-O distances are 2.211(2), 2.213(2), and 2.222(2) $\AA$, which compares favorably with the Sm-O bond lengths of 2.218(3), 2.222(4), and 2.232(4) $\AA$ in Sm(TRENDSAL) as well as the Nd-O distances of 2.241(6), 2.242(6), and 2.248(4) in Nd(TRENDSAL). [15a] Typical for complexes of this kind is the presence of two types of $\mathrm{Ln}-\mathrm{N}$ distances. In $\mathbf{1} \cdot \mathrm{DME}$ the average Eu-N distance to the three imine nitrogens is $2.526(2) \AA$, while a significantly longer Eu-N bond length of 2.793(2) $\AA$ is found for the interaction with the apical amine nitrogen. For comparison, in Sm(TRENDSAL) the corresponding Sm-N distances are 2.556(3) $\AA$ (average for imine nitrogens) and 2.818(4) A (apical). [15a]

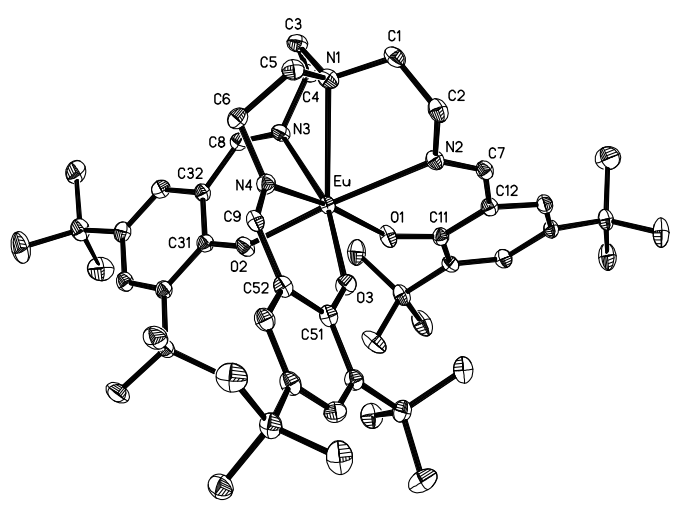

Figure 1. Molecular structure of $\mathrm{Eu}(\mathrm{TRENDSAL})$ in 1.DME.

A packing diagram (Figure 2) clearly shows that the DME solvent is just intercalated in the crystal structure as solvent of crystallization without any interaction with the europium. While this was not unexpected, we then investigated the crystallization of $\mathbf{1}$ from acetonitrile. It is well established from early work by Kanellakopulos et al. that the small, "slim" acetonitrile ligand is able to add to even fairly crowded lanthanide complexes such as the tris(cyclopentadienyls) [16] as well as the tris(amidinates). [17]

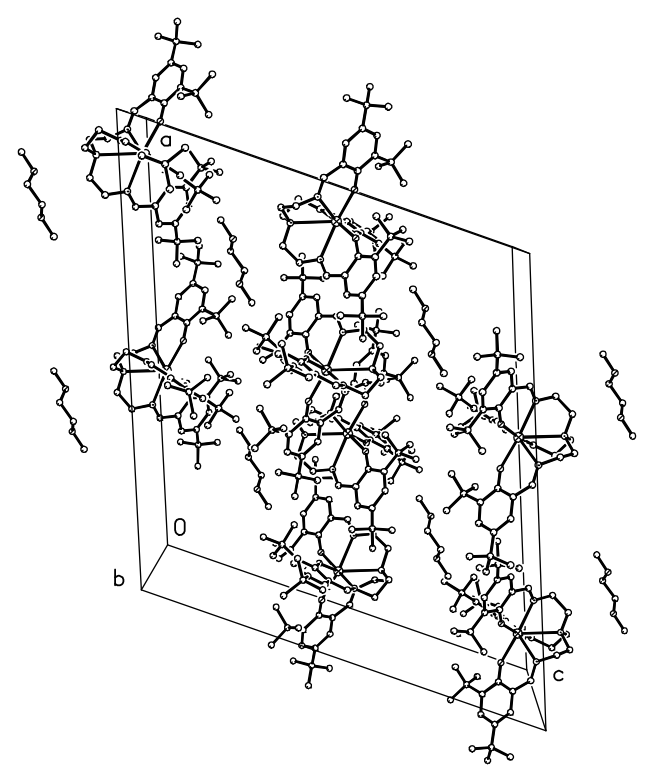

Figure 2. Unit cell of 1.DME showing the intercalated solvent of crystallization.

Crystallization of unsolvated $\mathbf{1}$ from acetonitrile afforded the solvate $\mathbf{1} \cdot \mathrm{MeCN}$ in the form of air-stable, bright yellow crystals. Once again, the X-ray study revealed the formation of a solvate with acetonitrile molecules intercalated in the crystal lattice of Eu(TRENDSAL) (Figure 3). This finding can be seen as a positive proof for the very effective encapsulation of the central europium(3+) ion by the bulky heptadentate TRENDSAL ligand. Clearly not even the "slim" acetonitrile ligand is able to enter the completely shielded coordination sphere of europium.

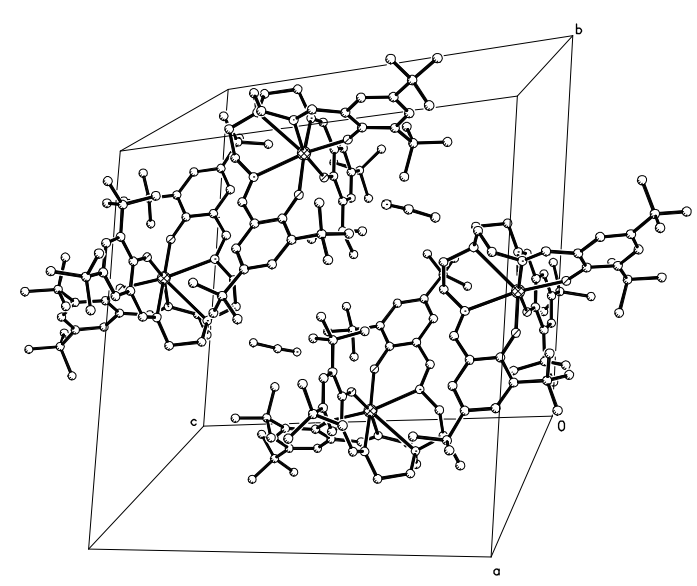

Figure 3. Unit cell of $\mathbf{1} \cdot \mathrm{MeCN}$ showing the intercalated solvent of crystallization. 
The solvate $\mathbf{1} \cdot \mathrm{MeCN}$ crystallizes in the triclinic space group P-1 with two independent molecules in the asymmetric unit (Table 1). As can been seen from Table 2, bond lengths and angles in the Eu(TRENDSAL) molecule in $\mathbf{1} \cdot \mathrm{MeCN}$ do not differ significantly from those in 1.DME. Apparently not even a labile complex exists in acetonitrile solution, as a ${ }^{1} \mathrm{H}$ NMR spectrum of $\mathbf{1}$ in $\mathrm{CD}_{3} \mathrm{CN}$ showed no measurable paramagnetic shift of the solvent resonances.

\section{Conclusions}

The new Schiff-base complex Eu(TRENDSAL) (1) was prepared in $71 \%$ yield in a straightforward manner by treatment of $\mathrm{EuCl}_{3} \cdot 6 \mathrm{H}_{2} \mathrm{O}$ simultaneously with 3,5-di-tertbutylsalicylicaldehyde and tris(2-aminoethyl)amine in the presence of triethylamine. Single crystal X-ray diffraction studies of the solvates $1 \cdot \mathrm{DME}$ and $1 \cdot \mathrm{MeCN}$ showed that encapsulation of the central $\mathrm{Eu}^{3+}$ ion by the bulky heptadentate TRENDSAL ligand is so effective that not even the small, "slim" acetonitrile ligand is able to enter the coordination sphere of europium.

\section{Experimental Section}

All starting materials were obtained from commercial sources and used as received. The ${ }^{1} \mathrm{H}$ NMR spectrum was recorded in THF- $d_{8}$ solution on a Bruker DPX 400 spectrometer at $25{ }^{\circ} \mathrm{C}$. Chemical shifts were referenced to TMS. Microanalysis was performed using a Leco CHNS 923 apparatus. The intensity data of $\mathbf{1} \cdot \mathrm{MeCN}$ and 1.DME were collected on a Stoe IPDS $2 \mathrm{~T}$ diffractometer with $\mathrm{MoK}_{\alpha}$ radiation. The data were collected with the Stoe XAREA [18] program using $\omega$-scans. Space groups were determined with XRED32 [18] program. The structures were solved by direct methods (SHELXS-97) and refined by full matrix least-squares methods on $F^{2}$ using SHELXL-97. [18]

Preparation Eu(TRENDSAL) (1): To a stirred solution of $2.93 \mathrm{~g}$ (8 mmol) europium(III) chloride hexahydrate in methanol $(100 \mathrm{ml})$ were added $5.60 \mathrm{~g}$ (24 mmol) 3,5-di-tert-butylsalicylicaldehyde in methanol $(80 \mathrm{ml})$ and $2.43 \mathrm{~g}(24 \mathrm{mmol})$ triethylamine in $80 \mathrm{ml}$ of the same solvent. The yellow mixture was stirred under reflux for 1 h. A solution of $1.30 \mathrm{~g}(8.9 \mathrm{mmol}$, slight excess $)$ of tris(2aminoethyl)amine in methanol $(80 \mathrm{ml})$ was added dropwise over a period of $30 \mathrm{~min}$ to the boiling reaction mixture, followed by refluxing for another $24 \mathrm{~h}$. The solvent was completely removed under reduced pressure using a rotary evaporator (300 mbar, $40{ }^{\circ} \mathrm{C}$ ) and desired Eu(TRENDSAL) (1) was purified by Soxhlet extraction of the remaining solid with $180 \mathrm{ml}$ of toluene. The clear, yellow extract was concentrated in vacuo to a volume of $50 \mathrm{ml}$ and cooled $5{ }^{\circ} \mathrm{C}$ for $24 \mathrm{~h}$ to afford $5.33 \mathrm{~g}(71 \%)$ of $\mathbf{1}$ as bright yellow crystals. Solvated crystals suitable for X-ray diffraction were obtained by recrystallization from either DME or acetonitrile. M.p. $234{ }^{\circ} \mathrm{C}$ (dec). IR (KBr pellet): $v_{\max } 2954$ (vs), 2905 (st), 2865 (m), 1619 (vs, C=N), 1548 (m), 1533 (st), 1460 (st), 1435 (st), 1412 (st), 1391 (st), 1361 (m), 1334 (st), 1325 (st), 1275 (m), 1257 (st), 1166 (st), $836(\mathrm{~m}), 744(\mathrm{st}) \mathrm{cm}^{-1} .{ }^{1} \mathbf{H}$ NMR $\left(400 \mathrm{MHz}, \mathrm{THF}-\mathrm{d}_{8}\right): \delta=-$ 29.78 (s, 3H, N=CH), -0.68 (s, 3H, Ar-H), -0.28 (s, 27H, $\left.{ }^{\mathrm{t}} \mathrm{Bu}\right), 3.23$ (s, 3H, Ar-H), 3.39 (s, 3H, $\left.\mathrm{CH}_{2}\right), 7.82\left(\mathrm{~s}, 27 \mathrm{H},{ }^{\mathrm{t}} \mathrm{Bu}\right), 11.52$ (s br, $\left.3 \mathrm{H}, \mathrm{CH}_{2}\right), 16.74\left(\mathrm{~s}, 3 \mathrm{H}, \mathrm{CH}_{2}\right), 28.88\left(\mathrm{~s} \mathrm{br}, 3 \mathrm{H}, \mathrm{CH}_{2}\right)$. EI-MS: $\mathrm{m} / \mathrm{z}$ $944.7\left(90,[\mathrm{M}]^{+}\right), 929.7\left(20,\left[\mathrm{M}-\mathrm{CH}_{3}\right]^{+}\right), 219.1(100,[\mathrm{CH}(2-\mathrm{OH}-$ $\left.\left.\left.3,5-{ }^{t} \mathrm{Bu}_{2} \mathrm{C}_{6} \mathrm{H}_{2}\right)\right]^{+}\right)$.
Supporting Information (see footnote on the first page of this article): ORTEP drawings and X-ray structural data as well as complete CIF files for $\mathbf{1} \cdot \mathbf{D M E}$ and $\mathbf{1} \cdot \mathbf{M e C N}$.

Table 1. Crystallographic Data for $\mathbf{1} \cdot \mathrm{MeCN}$ and $1 \cdot \mathrm{DME}$.

\begin{tabular}{|c|c|c|}
\hline & 1.DME & $\mathbf{1} \cdot \mathrm{MeCN}$ \\
\hline Empirical formula & $\mathrm{C}_{53} \mathrm{H}_{83} \mathrm{EuN}_{4} \mathrm{O}_{4}$ & $\mathrm{C}_{53} \mathrm{H}_{78} \mathrm{EuN}_{5} \mathrm{O}_{3}$ \\
\hline$a(\AA)$ & $27.648(6)$ & $16.3739(2)$ \\
\hline$b(\AA)$ & $16.227(3)$ & $17.8199(2)$ \\
\hline$c(\AA)$ & $24.817(5)$ & 20.7191(3) \\
\hline$\alpha\left(^{\circ}\right)$ & 90 & $93.0927(11)$ \\
\hline$\beta\left(^{\circ}\right)$ & 111.71(3) & $109.0384(11)$ \\
\hline$\gamma\left({ }^{\circ}\right)$ & 90 & $112.2090(11)$ \\
\hline$V\left(\AA^{3}\right)$ & $10344(4)$ & $5181.13(13)$ \\
\hline 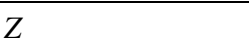 & 8 & 4 \\
\hline Formula weight & 992.19 & 985.16 \\
\hline Space group & $\mathrm{C} 2 / \mathrm{c}$ & $\mathrm{P}-1$ \\
\hline$T\left({ }^{\circ} \mathrm{C}\right)$ & -140 & -100 \\
\hline$\lambda(\AA)$ & 0.71073 & 0.71073 \\
\hline $\mathrm{D}_{\text {calcd }}\left(\mathrm{g} \mathrm{cm}^{-3}\right)$ & 1.274 & 1.263 \\
\hline$\mu\left(\mathrm{mm}^{-1}\right)$ & 1.258 & 1.255 \\
\hline $\begin{array}{l}\text { Independent } \\
\text { reflections }\end{array}$ & 12770 & 27967 \\
\hline Parameters & 578 & 1156 \\
\hline Largest diff. peak & 2.421 & 0.897 \\
\hline $\mathrm{R}\left(\mathrm{Fo}\right.$ or $\left.\mathrm{Fo}^{2}\right)$ & 0.0395 & 0.0486 \\
\hline $\mathrm{R}_{\mathrm{w}}\left(\mathrm{Fo}\right.$ or $\left.\mathrm{Fo}^{2}\right)$ & 0.0955 & 0.0708 \\
\hline
\end{tabular}

Table 2. Bond lengths $[\AA]$ and angles $\left[{ }^{\circ}\right]$ for $\mathbf{1} \cdot \mathrm{DME}$.

\begin{tabular}{|lr|lr|}
\hline Eu-O(1) & $2.211(2)$ & Eu-N(2) & $2.531(2)$ \\
$\mathrm{Eu}-\mathrm{O}(2)$ & $2.213(2)$ & $\mathrm{Eu}-\mathrm{N}(1)$ & $2.793(2)$ \\
$\mathrm{Eu}-\mathrm{O}(3)$ & $2.222(2)$ & $\mathrm{N}(2)-\mathrm{C}(7)$ & $1.287(4)$ \\
$\mathrm{Eu}-\mathrm{N}(4)$ & $2.516(2)$ & $\mathrm{N}(3)-\mathrm{C}(8)$ & $1.290(4)$ \\
$\mathrm{Eu}-\mathrm{N}(3)$ & $2.530(2)$ & $\mathrm{N}(4)-\mathrm{C}(9)$ & $1.283(4)$ \\
& & & \\
\hline $\mathrm{O}(1)-\mathrm{Eu}-\mathrm{O}(2)$ & $95.08(8)$ & $\mathrm{O}(2)-\mathrm{Eu}-\mathrm{N}(2)$ & $164.97(8)$ \\
$\mathrm{O}(1)-\mathrm{Eu}-\mathrm{O}(3)$ & $96.13(8)$ & $\mathrm{O}(3)-\mathrm{Eu}-\mathrm{N}(2)$ & $91.64(8)$ \\
$\mathrm{O}(2)-\mathrm{Eu}-\mathrm{O}(3)$ & $94.61(8)$ & $\mathrm{N}(4)-\mathrm{Eu}-\mathrm{N}(2)$ & $105.23(8)$ \\
$\mathrm{O}(1)-\mathrm{Eu}-\mathrm{N}(4)$ & $166.90(8)$ & $\mathrm{N}(3)-\mathrm{Eu}-\mathrm{N}(2)$ & $103.56(8)$ \\
$\mathrm{O}(2)-\mathrm{Eu}-\mathrm{N}(4)$ & $89.73(8)$ & $\mathrm{O}(1)-\mathrm{Eu}-\mathrm{N}(1)$ & $120.75(7)$ \\
$\mathrm{O}(3)-\mathrm{Eu}-\mathrm{N}(4)$ & $71.30(8)$ & $\mathrm{O}(2)-\mathrm{Eu}-\mathrm{N}(1)$ & $121.34(8)$ \\
$\mathrm{O}(1)-\mathrm{Eu}-\mathrm{N}(3)$ & $89.53(8)$ & $\mathrm{O}(3)-\mathrm{Eu}-\mathrm{N}(1)$ & $122.23(7)$ \\
$\mathrm{O}(2)-\mathrm{Eu}-\mathrm{N}(3)$ & $70.78(7)$ & $\mathrm{N}(4)-\mathrm{Eu}-\mathrm{N}(1)$ & $65.92(7)$ \\
$\mathrm{O}(3)-\mathrm{Eu}-\mathrm{N}(3)$ & $164.79(8)$ & $\mathrm{N}(3)-\mathrm{Eu}-\mathrm{N}(1)$ & $65.37(7)$ \\
$\mathrm{N}(4)-\mathrm{Eu}-\mathrm{N}(3)$ & $103.56(8)$ & $\mathrm{N}(2)-\mathrm{Eu}-\mathrm{N}(1)$ & $65.49(8)$ \\
$\mathrm{O}(1)-\mathrm{Eu}-\mathrm{N}(2)$ & $70.64(8)$ & & \\
& & & \\
\hline
\end{tabular}


Table 3. Bond lengths $[\AA ̊]$ and angles $\left[^{\circ}\right]$ for $\mathbf{1} \cdot \mathrm{MeCN}$.

\begin{tabular}{|c|c|c|c|}
\hline $\mathrm{Eu}(1)-\mathrm{O}(1)$ & $2.1901(17)$ & $\mathrm{Eu}(2)-\mathrm{O}(13)$ & $2.2114(17)$ \\
\hline $\mathrm{Eu}(1)-\mathrm{O}(2)$ & $2.2212(16)$ & $\mathrm{Eu}(2)-\mathrm{O}(11)$ & $2.2151(17)$ \\
\hline $\mathrm{Eu}(1)-\mathrm{O}(3)$ & $2.2219(17)$ & $\mathrm{Eu}(2)-\mathrm{O}(12)$ & $2.2172(16)$ \\
\hline $\mathrm{Eu}(1)-\mathrm{N}(3)$ & $2.513(2)$ & $\mathrm{Eu}(2)-\mathrm{N}(14)$ & $2.507(2)$ \\
\hline $\mathrm{Eu}(1)-\mathrm{N}(4)$ & $2.526(2)$ & $\mathrm{Eu}(2)-\mathrm{N}(13)$ & $2.5194(19)$ \\
\hline $\mathrm{Eu}(1)-\mathrm{N}(2)$ & $2.543(2)$ & $\mathrm{Eu}(2)-\mathrm{N}(12)$ & $2.534(2)$ \\
\hline $\mathrm{Eu}(1)-\mathrm{N}(1)$ & $2.789(2)$ & $\mathrm{Eu}(2)-\mathrm{N}(11)$ & $2.806(2)$ \\
\hline $\mathrm{N}(2)-\mathrm{C}(7)$ & $1.284(3)$ & $\mathrm{N}(12)-\mathrm{C}(107)$ & $1.280(3)$ \\
\hline $\mathrm{N}(3)-\mathrm{C}(8)$ & $1.282(3)$ & $\mathrm{N}(13)-\mathrm{C}(108)$ & $1.279(3)$ \\
\hline $\mathrm{N}(4)-\mathrm{C}(9)$ & $1.280(3)$ & $\mathrm{N}(14)-\mathrm{C}(109)$ & $1.281(3)$ \\
\hline $\mathrm{O}(1)-\mathrm{Eu}(1)-\mathrm{O}(2)$ & $95.57(6)$ & $\mathrm{O}(13)-\mathrm{Eu}(2)-\mathrm{O}(11)$ & $99.12(6)$ \\
\hline $\mathrm{O}(1)-\mathrm{Eu}(1)-\mathrm{O}(3)$ & $92.85(7)$ & $\mathrm{O}(13)-\mathrm{Eu}(2)-\mathrm{O}(12)$ & $94.14(7)$ \\
\hline $\mathrm{O}(2)-\mathrm{Eu}(1)-\mathrm{O}(3)$ & $94.91(6)$ & $\mathrm{O}(11)-\mathrm{Eu}(2)-\mathrm{O}(12)$ & $96.65(6)$ \\
\hline $\mathrm{O}(1)-\mathrm{Eu}(1)-\mathrm{N}(3)$ & $91.44(7)$ & $\mathrm{O}(13)-\mathrm{Eu}(2)-\mathrm{N}(14)$ & $71.56(6)$ \\
\hline $\mathrm{O}(2)-\mathrm{Eu}(1)-\mathrm{N}(3)$ & $70.90(6)$ & $\mathrm{O}(11)-\mathrm{Eu}(2)-\mathrm{N}(14)$ & $169.72(7)$ \\
\hline $\mathrm{O}(3)-\mathrm{Eu}(1)-\mathrm{N}(3)$ & $165.52(7)$ & $\mathrm{O}(12)-\mathrm{Eu}(2)-\mathrm{N}(14)$ & $88.51(7)$ \\
\hline $\mathrm{O}(1)-\mathrm{Eu}(1)-\mathrm{N}(4)$ & $162.66(7)$ & $\mathrm{O}(13)-\mathrm{Eu}(2)-\mathrm{N}(13)$ & $164.13(7)$ \\
\hline $\mathrm{O}(2)-\mathrm{Eu}(1)-\mathrm{N}(4)$ & $91.49(7)$ & $\mathrm{O}(11)-\mathrm{Eu}(2)-\mathrm{N}(13)$ & $87.39(7)$ \\
\hline $\mathrm{O}(3)-\mathrm{Eu}(1)-\mathrm{N}(4)$ & $70.74(7)$ & $\mathrm{O}(12)-\mathrm{Eu}(2)-\mathrm{N}(13)$ & $70.63(6)$ \\
\hline $\mathrm{N}(3)-\mathrm{Eu}(1)-\mathrm{N}(4)$ & $105.84(7)$ & $\mathrm{N}(14)-\mathrm{Eu}(2)-\mathrm{N}(13)$ & $102.73(7)$ \\
\hline $\mathrm{O}(1)-\mathrm{Eu}(1)-\mathrm{N}(2)$ & $70.35(6)$ & $\mathrm{O}(13)-\mathrm{Eu}(2)-\mathrm{N}(12)$ & 91.61(7) \\
\hline $\mathrm{O}(2)-\mathrm{Eu}(1)-\mathrm{N}(2)$ & $165.02(7)$ & $\mathrm{O}(11)-\mathrm{Eu}(2)-\mathrm{N}(12)$ & $70.79(6)$ \\
\hline $\mathrm{O}(3)-\mathrm{Eu}(1)-\mathrm{N}(2)$ & $90.99(7)$ & $\mathrm{O}(12)-\mathrm{Eu}(2)-\mathrm{N}(12)$ & $166.90(7)$ \\
\hline $\mathrm{N}(3)-\mathrm{Eu}(1)-\mathrm{N}(2)$ & $103.47(6)$ & $\mathrm{N}(14)-\mathrm{Eu}(2)-\mathrm{N}(12)$ & $104.48(7)$ \\
\hline $\mathrm{N}(4)-\mathrm{Eu}(1)-\mathrm{N}(2)$ & $103.47(7)$ & $\mathrm{N}(13)-\mathrm{Eu}(2)-\mathrm{N}(12)$ & $104.22(7)$ \\
\hline $\mathrm{O}(1)-\mathrm{Eu}(1)-\mathrm{N}(1)$ & $122.28(7)$ & $\mathrm{O}(13)-\mathrm{Eu}(2)-\mathrm{N}(11)$ & $122.41(6)$ \\
\hline $\mathrm{O}(2)-\mathrm{Eu}(1)-\mathrm{N}(1)$ & $121.47(6)$ & $\mathrm{O}(11)-\mathrm{Eu}(2)-\mathrm{N}(11)$ & $118.33(7)$ \\
\hline $\mathrm{O}(3)-\mathrm{Eu}(1)-\mathrm{N}(1)$ & $122.43(7)$ & $\mathrm{O}(12)-\mathrm{Eu}(2)-\mathrm{N}(11)$ & $120.48(6)$ \\
\hline $\mathrm{N}(3)-\mathrm{Eu}(1)-\mathrm{N}(1)$ & $65.82(6)$ & $\mathrm{N}(14)-\mathrm{Eu}(2)-\mathrm{N}(11)$ & $65.62(6)$ \\
\hline $\mathrm{N}(4)-\mathrm{Eu}(1)-\mathrm{N}(1)$ & $65.83(7)$ & $N(13)-E u(2)-N(11)$ & $65.18(6)$ \\
\hline $\mathrm{N}(2)-\mathrm{Eu}(1)-\mathrm{N}(1)$ & $65.52(6)$ & $\mathrm{N}(12)-\mathrm{Eu}(2)-\mathrm{N}(11)$ & $65.20(6)$ \\
\hline
\end{tabular}

\section{Acknowledgement}

P. D. thanks the government of Sachsen-Anhalt for a Ph.D. scholarship (Graduiertenförderung). Financial support by the Ottovon-Guericke-Universität Magdeburg is also gratefully acknowledged.

[1] a) R.B. Lauffer, Chem. Rev. 1987, 87, 901; b) P. Caravan, J.J. Ellison, T.J. McMurry, R.B. Lauffer, Chem. Rev. 1999, 99, 2293.

[2] a) O. Kocian, K.W. Chiu, R. Demeure,B. Gallez, C.J. Jones, J.R. Thornback, J. Chem. Soc., Perkin Trans. 1994, 527; b) S.W.A. Bligh, N. Choi, E.G. Evagorou, M. McPartlin, W.J. Cummins, J.D. Kelly, Polyhedron 1992, 11, 2571; c) P.H. Smith, J.R. Brainard, D.E. Morris, G.D. Jarvinen, R.R. Ryan, J. Am. Chem. Soc. 1989, 111, 7437.

[3] a) M.E. Marmion, S.R. Woulfe, W.L. Newmann, G. Pilcher, D.L. Nosco, Nucl. Med. Biol. 1996, 23, 567; b) Y. Coulais, G. Cros, M.H. Darbieu, J.A.M. Tafani, H. Belhadj-Tahar, E.

Bellande, R. Pasqualini, R. Guiraud, Nucl. Med. Biol. 1994, 21, 263; c) E.K. John, A.J. Bott, M.A. Green, J. Pharm. Sci. 1994, 83, 587.

[4] a) P. Bhattacharyya, J. Parr, A.M.Z. Slawin, Inorg. Chem. Commun. 1999, 2, 113; b) M.R.A. Pillai, K. Kothari, B. Mathew, N.K. Pilkwal, S. Jurisson, Nucl. Med. Biol. 1999, 26, 233; c) P. Wei, D.A. Atwood, J. Organomet. Chem. 1998, 563, 87; d) J. Parr, A.T. Ross, A.M.Z. Slawin, Inorg. Chem. Commun. 1998, 1, 59; e) P.K. Bharadwaj, A.M. Lee, S. Mandal, B.W. Skelton, A.H. White, Aust. J. Chem. 1994, 47, 1799; f) S. Liu, S.J. Rettig, C. Orvig, Inorg. Chem. 1992, 31, 5400 .

[5] S. Liu, L-W. Yang, S.J. Rettig, C. Orvig, Inorg. Chem. 1993, 32, 2773.

[6] P. Caravan, T. Hedlund, S. Liu, S. Sjöberg, C. Orvig, J. Am. Chem. Soc. 1995, 117, 11230.

[7] J.-P. Costes, F. Dahan, A. Dupuis, S. Lagrave, J.-P. Laurent, Inorg. Chem. 1998, 37, 153.

[8] U. Casellato, S. Tamburini, P. Tomasin, P.A. Vigato, M. Botta, Inorg. Chim. Acta 1996, 247, 143.

[9] J.-P. Costes, A. Dupuis, G. Commenges, S. Lagrave, J.-P. Laurent, Inorg. Chim. Acta 1999, 285, 49.

[10] D.J. Berg, S.J. Rettig, C. Orvig, J. Am. Chem. Soc. 1991, 113, 2528.

[11] M. Kanesato, T. Yokoyama, Chem. Lett. 1999, 137.

[12] M. Kanesato, T. Yokoyama, O. Itabashi, T.M. Suzuki, M. Shiro, Bull. Chem. Soc. Jpn. 1996, 69, 1297.

[13] a) A. Malek, G.C. Dey, A. Nasreen, T.A. Chowdhury, E.C. Alyea, Synth. React. Inorg. Met.-Org. Chem. 1979, 9, 145; b) K. Ramesh, R. Mukherjee, J. Chem. Soc., Dalton Trans. 1991, 3259; c) A. Aguiari, E. Bullita, U. Casellato, P. Guerriero, S. Tamburini, P.A. Vigato, U. Russo, Inorg. Chim. Acta 1994, 219, 135.

[14] a) G.C. De, Nucl. Sci. Appl., Ser. B 1983, 59; b) S. Liu, L. Gelmini, S.J. Rettig, R.C. Thompson, C. Orvig, J. Am. Chem. Soc. 1992, 114, 6081; c) J.-P. Costes, A. Dupuis, J.-P. Laurent, Eur. J. Inorg. Chem. 1998, 1543; d) N. Brianese, U. Casellato, S. Tamburini, P. Tomasin, P.A. Vigato, Inorg. Chim. Acta 1998, 272, 235.

[15] a) M. W. Essig, W. Keogh, B. L. Scott, J. G. Watkin, Polyhedron 2001, 20, 373; b) S. Salehzadeh, S. M. Nouri, H. Keypour, M. Bagherzadeh, Polyhedron 2005, 24, 1478; c) J. Parr, A. T. Ross, A. M. Z. Slawin, Main Group Chem. 1998, $4,243$.

[16] a) L. Xing-Fu, S. Eggers, J. Kopf, W. Jahn, R. D. Fischer, C. Apostolidis, B. Kanellakopulos, F. benetollo, A. Polo, G. Bombieri, Inorg. Chim. Acta 1985, 100, 183; b) M. R. Spirlet, J. Rebizant, C. Apostolidis, B. Kanellakopulos, Inorg. Chim. Acta 1987, 139, 211.

[17] J. Richter, J. Feiling, H.-G. Schmidt, M. Noltemeyer, W. Brüser, F.T. Edelmann, Z. Anorg. Allg. Chem. 2004, 630, 1269.

[18] Stoe, XAREA Program for Xray Crystal Data collection, (XRED32 included in XAREA) (Stoe, 2002)

[19] a) G. M. Sheldrick, SHELXL-97 Program for Crystal Structure Refinement, Universität Göttingen, Germany, 1997; b) G. M. Sheldrick, SHELXS-97 Program for Crystal Structure Solution, Universität Göttingen, Germany, 1997.

Received: ((will be filled in by the editorial staff)) Published online: ((will be filled in by the editorial staff)) 
Entry for the Table of Contents

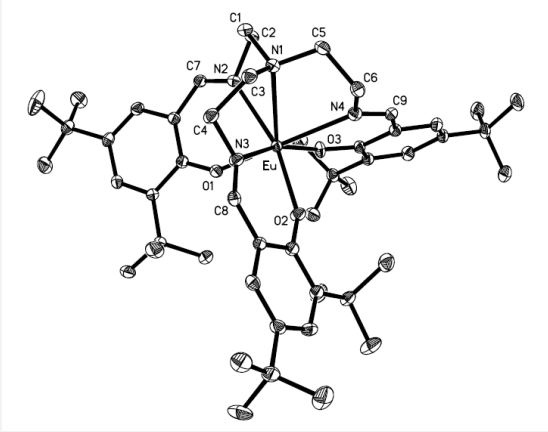

Effective Encapsulation of Europium(III) by a Heptadentate Schiff-Base Ligand

Peter Dröse, Cristian G. Hrib and Frank T. Edelmann*

Page No. - Page No. 